Palavras chave: Fitossociologia

Áreas carsticas

Florestas deciduais

Histórico:

Recebido 03/10/2012

Aceito 28/0I/2015

Keywords:

Phytosociology

Karst areas

Seasonally Dry Tropical Forests

Correspondência: giselecristina.biologa@yahoo.com.br

DOI: I0.1590/01047760201521021609
Gisele Cristina de Oliveira Menino', Rubens Manoel dos Santos², Deborah Mattos Guimarães Apgaua², Gabriela Gomes Pires², Diego Gualberto Sales Pereira², Marco Aurélio Leite Fontes ${ }^{2}$, Hisaias de Souza Almeida ${ }^{3}$

\section{FLORÍSTICA E ESTRUTURA DE FLORESTAS TROPICAIS SAZONALMENTE SECAS}

RESUMO: Neste estudo, objetivou-se caracterizar florística e estruturalmente a comunidade arbórea em uma área de encosta, Caatinga arbórea e em área ecotónal com cerrado no Parque Nacional Cavernas do Peruaçu, Norte de Minas Gerais, buscando testar as seguintes hipóteses: I - Existem diferenciações florísticas entre os ambientes; 2- Todos os ambientes apresentam as mesmas características quanto à estrutura. Foram confeccionados diagrama de Venn e histogramas de frequência da distribuição de indivíduos por classes de diâmetro. Foi feita a análise de Espécies Indicadoras e foram calculados os parâmetros fitossociológicos clássicos. Foram encontradas 305 espécies, distribuídas em 173 gêneros e 48 famílias. Sendo que, na encosta, foram encontradas 166 espécies, na Caatinga arbórea foram encontradas 204 espécies e na área ecotonal foram encontradas 155 espécies. Muitas espécies foram exclusivas a cada ambiente, sendo 54 à encosta, 65 à Caatinga arbórea e 35 ao ecótono. Apenas 69 espécies foram comuns aos três ambientes. Foram amostrados 139I indivíduos, resultando em uma estimativa de 1340,62, I765 e 1280 indivíduos ha $^{-1}$ na encosta, Caatinga arbórea e ecótono, respectivamente. $A$ área basal em $\mathrm{m}^{2} \cdot$ ha $^{-1}$ foi 47,64, 30,05 e 23,29, respectivamente. Já, o índice de diversidade de Shannon foi 3,49, 3,74 e 3,6I nats-indivíduo-1 e a equabilidade de Pielou foi de 0,80, 0,84 e 0,85 . Com relação à distribuição dos indivíduos em classes de diâmetro, a Caatinga arbórea e encosta apresentaram um mesmo padrão, com uma diminuição gradual do número de indivíduos. Já, o ambiente ecotonal apresentou uma distribuição tendendo a normal.

\section{FLORISTIC AND STRUCTURE OF SEASONALLY DRY TROPICAL FORESTS}

ABSTRACT: A floristic and structural survey of the tree community in different environments (slope, Arboreal Caatinga and ecotone to savannah) were carried out in Cavernas do Peruaçu National Park, North of Minas Gerais. We aimed to test the following hypotheses: I - There is floristic differentiation among the environments; 2 All environments have the same structural characteristics. Venn Diagrams were used to compare both the shared and unshared species among the environments. Tree distribution by diameter classes for each environment was made by means of frequency histograms. An Indicator Species Analysis and usual phytossociological parameters were used. A total of 305 species were found, distributed in 173 genera and 48 families, of which 166 were found in the slope, 204 in the Arboreal Caatinga and 155 in the ecotone. 54 species were exclusive to the slope, 65 to the Arboreal Caatinga and 35 to the ecotone and only 69 species were common to all three environments. 1391 individuals were sampled, yielding

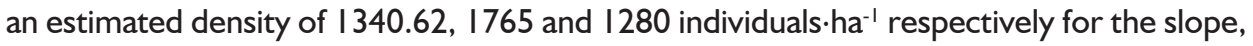
Arboreal Caatinga and ecotone. The basal area reached 47.64, 30.05 and $23.29 \mathrm{~m}^{2} \cdot \mathrm{ha}^{-1}$, respectively for the slope, Arboreal Caatinga and ecotone. The Shannon diversity index and Pielou's evenness were, respectively, 3.49 and 0.80 for the slope, 3.74 and 0.84 for the Arboreal Caatinga and 3.61 and 0.85 for the ecotone. The Arboreal Caatinga and the slope presented a gradual decrease in frequency towards the higher diameter classes whereas the ecotone tended to follow a normal distribution.

I Instituto Federal Goiano - Rio Verde, Goiás, Brasil

2 Universidade Federal de Lavras - Lavras, Minas Gerais, Brasil

${ }^{3}$ Universidade Federal de Itajubá - Itajubá, Minas Gerais, Brasil 


\section{INTRODUÇÃO}

As Florestas Tropicais Sazonalmente Secas (FTSS) são encontradas do noroeste do México, ao norte da Argentina e sudoeste do Brasil em áreas disjuntas de tamanhos variados (LINNARES-PALLOMINO et al., 20I I).

A teoria do Arco Pleistocênico propõe que - padrão atual de distribuição das FTSS representa as relíquias de uma formação contígua que atingiu sua extensão máxima durante os períodos de contração das florestas úmidas (LINNARES-PALLOMINO et al., 20ll). A extensão contínua das FTSS se estendia desde a Caatinga brasileira para as áreas periféricas do domínio Chaco, possivelmente, também atingindo os vales secos interandinos da Bolívia, Peru e Equador (PRADO; GIBBS, 1993). Essa formação é encontrada dentro de um complexo de tipos de vegetação, dependendo das condições locais de solo, clima e condições topográficas (PENNINGTON et al., 2009).

No Norte do Estado de Minas Gerais, encontrase a fronteira sul para o bioma da Caatinga (QUEIROZ, 2006). Essa região é um complexo vegetacional, sendo uma área de transição entre os Domínios da Caatinga e do Cerrado, suportando fitofisionomias bem diferenciadas, principalmente no que se refere à composição de espécies e à abundância e porte dos indivíduos (SANTOS et al., 2007).

A floresta estacional decidual no norte de Minas ainda é pouco estudada e essa região destacase por ser uma área de prioridade para a conservação da diversidade biológica da Caatinga (TABARELLI; SILVA, 2003). Contudo, apesar da baixa prioridade de conservação que é dada para a Caatinga, o bioma contém maior riqueza de espécies e maior endemismo do que é geralmente admitido (OLIVEIRA et al., 20I2). Apesar dessas particularidades, a Caatinga arbórea no Norte de Minas Gerais vem sofrendo intensa exploração dos recursos naturais, principalmente para dar lugar à prática da agropecuária e à extração de madeira para carvão (SANTOS et al., 20I I).

Além disso, o Parque Nacional Cavernas do Peruraçu constitui-se de uma área cárstica e são raros os trabalhos de levantamento florístico e fitossociologicos nessas áreas, contudo levantamentos fitossociológicos como os de Santos et al. (2007, 20I I) têm revelado a riqueza e a diversidade da comunidade arbórea em florestas estacionais deciduais no norte de Minas.

Dessa forma, no presente estudo, objetivouse caracterizar a florística e a estrutura da comunidade arbórea em três ambientes de um fragmento de Floresta
Tropical Sazonalmente Seca no Parque Nacional Cavernas do Peruaçu, Norte de Minas Gerais, buscando testar as seguintes hipóteses: I- Existem diferenciações florísticas entre os ambientes; 2- Todos os ambientes apresentam as mesmas características quanto à estrutura.

\section{MATERIAL E MÉTODOS}

\section{Área de estudo}

O estudo foi desenvolvido no Parque Nacional Cavernas do Peruaçu, com 56800 ha, localizado a $15^{\circ} 06^{\prime} 38^{\prime}$ 'S, 44I.'52”W (VELLOSO et al., 2002), nos municípios mineiros de Itacarambi, Januária e São João das Missões. O Parque foi criado em 1999 (BRASIL, 1999). Essa área está incluída no semiárido brasileiro, apresentando uma precipitação média anual menor que $800 \mathrm{~mm}$, alta evapotranspiração potencial e índice de aridez de 0.5 , ou um risco de seca maior que $60 \%$ (BRASIL, 2005).

As formações florestais encontradas no Peruaçu são: Floresta Decidual Estacional Montana, Floresta Estacional Semidecidual, Savana Arborizada e áreas de tensão ecológica entre Savana Estépica e Floresta Estacional. Por se tratar de uma região de transição entre dois biomas, a UC tem grande diversidade e complexidade de ambientes (GOMES et al., 2007).

\section{Coleta dos dados}

Como a área de estudo apresenta uma grande diversidade de ambientes, parcelas foram alocadas em alguns trechos com variações ambientais marcantes, com o intuito de caracterizar a estrutura florestal de ambientes distintos. $\mathrm{Na}$ área caracterizada como encosta do afloramento de calcário foram alocadas 8 parcelas de $20 \times 20 \mathrm{~m}$, sendo estas o grupo de parcelas mais úmidas. $\mathrm{Na}$ área caracterizada como Caatinga arbórea (SANTOS et al., 20I2), que compreendeu mata sob solo profundo sem rocha aflorada e mata sobre afloramento calcário, foram alocadas 10 parcelas e na área ecotonal com o cerrado foram alocadas 5 parcelas. $O$ número de parcelas inferior a dez, em duas áreas, deve-se ao pequeno tamanho da área com essas características. A amostragem ocorreu nos meses de janeiro e julho de 201 I. Em cada parcela foram amostrados todos os indivíduos arbóreos com circunferência a I,30 m acima do solo (CAP) maior ou igual a $10 \mathrm{~cm}$. Os indivíduos foram marcados com plaquetas de alumínio numeradas e tiveram a altura estimada. 
Já, o levantamento florístico foi realizado por meio de caminhadas aleatórias semestrais no período de janeiro de 2003 à abril de 2012, ao longo do Parque Nacional Cavernas do Peruaçu no intuito de catalogar as espécies presentes.

\section{Análise dos dados}

Para análise da similaridade florística entre os grupos, foi confeccionado um diagrama de Venn, com base na presença e ausência das espécies, permitindo avaliar a conexão florística entre os ambientes (OLIVEIRA FILHO; RATTER, 2009), evidenciando o número de espécies exclusivas e comuns aos três ambientes.

Foram calculados os parâmetros fitossociológicos clássicos: densidade, dominância e frequência, absolutas e relativas e o valor de importância (MUELLER-DOMBOIS; ELLEMBERG, 1974). O índice de diversidade de Shannon e a equabilidade de Pielou foram calculados (BROWER; ZAR, 1984). Histogramas de frequência da distribuição de indivíduos por classes de diâmetro foram confeccionados, para verificar diferenças entre os ambientes.

A Análise de Espécies Indicadoras (DUFRÊNE; LEGENDRE, 1997) foi utilizada para avaliar quais espécies eram características de cada ambiente amostrado. $O$ valor indicador (Vallnd) foi comparado aos resultados de 1000 aleatorizações de dados, usando o teste de Monte Carlo. Esse método, combina a informação sobre a concentração da abundância de uma espécie em certo grupo de unidades amostrais e sobre a fidelidade da ocorrência dessa espécie a esse mesmo grupo. Os cálculos também foram processados pelo programa PC-Ord for Windows versão 4. I4 (MCCUNE; MEFFORD, 1999).

\section{RESULTADOS E DISCUSSÃO}

\section{Florística}

Foram encontradas 305 espécies arbustivas/ arbóreas, distribuídas em 173 gêneros e 48 famílias (Tabela I), sendo que, na encosta, foram encontradas 166 espécies, na Caatinga arbórea foram 204 espécies e na área ecotonal 155 espécies. Muitas espécies foram exclusivas a cada ambiente, sendo 54 à encosta, 65 à Caatinga arbórea e 35 ao ecotono. Apenas 69 espécies foram comuns aos três ambientes (Figura I). Esses números evidenciam a grande heterogeneidade da área estudada, com uma composição de espécies própria, realçando sua importância para a conservação da biodiversidade.
TABELA 1 Espécies encontradas no Parque Nacional do Peruaçu, em seus respectivos ambientes, sendo: 1= Encosta; 2= Caatinga arbórea; $3=$ Ecótono com cerrado.

TABLE 1 Species found in Peruaçu National Park in each environment, in which: $1=$ Slope; $2=$ Arboreal Caatinga, $3=$ Savannah ecotone.

\begin{tabular}{|c|c|c|c|}
\hline \multirow{2}{*}{ Família/Espécie } & \multicolumn{3}{|c|}{ Ambiente } \\
\hline & 1 & 2 & 3 \\
\hline \multicolumn{4}{|l|}{ Anacardiaceae } \\
\hline Astronium fraxinifolium Schott ex Spreng. & $x$ & $x$ & $x$ \\
\hline Cyrtocarpa caatingae J.D.Mitch. \& Daly & $x$ & $x$ & \\
\hline Myracrodruon urundeuva Allemão & $x$ & $x$ & $x$ \\
\hline Schinopsis brasiliensis Engl. & $x$ & $x$ & $\mathrm{x}$ \\
\hline Spondias mombin L. & $x$ & $x$ & $x$ \\
\hline Spondias tuberosa Arruda & $x$ & $x$ & \\
\hline \multicolumn{4}{|l|}{ Annonaceae } \\
\hline Annona leptopetala (R.E.Fr.) H.Rainer & & $\mathrm{x}$ & $x$ \\
\hline Annona vepretorum Mart. & & $x$ & \\
\hline Rollinia dolabripetala (Raddi) R.E.Fr. & & $x$ & \\
\hline Rollinia sylvatica (A.St.-Hil.) Mart. & & & $x$ \\
\hline
\end{tabular}

Apocynaceae

Aspidosperma cuspa (Kunth) S.F.Blake ex Pittier $\quad x \quad x \quad x$

Aspidosperma cylindrocarpon Müll.Arg.

Aspidosperma multiflorum A. DC.

$x \quad x$

Aspidosperma polyneuron Müll.Arg.

$x \quad x$

Aspidosperma pyrifolium Mart.

$x \quad x$

X $\quad x \quad x$

Aspidosperma subincanum Mart. ex A.DC. $\quad \mathrm{x}$

Araliaceae

Aralia excelsa (Griseb.) J. Wen

$x \quad x$

Aralia warmingiana (Marchal) J.Wen

$x \quad x$

Arecaceae

Acrocomia aculeata (Jacq.) Lodd. ex Mart. $\quad \mathrm{x}$

Syagrus oleracea (Mart.) Becc.

$\mathrm{X} \times$

Asteraceae

Baccharis semiserrata (Steud.) G.M.Barroso

$x$

Dasyphyllum brasiliense (Spreng.) Cabrera $\quad \mathrm{x}$

Piptocarpha rotundifolia (Less.) Baker

$x$

Vernonanthura divaricata (Spreng.) H.Rob. $\quad$ x

Vernonanthura phosphorica (Vell.) H.Rob. x

Bignoniaceae

Fridericia bahiensis (Schauer ex. DC.)

L.G.Lohmann

$\mathrm{x}$

Handroanthus heptaphyllus (Martius) Mattos $\quad x \quad x$

Handroanthus impetiginosus (Mart. ex DC.) Mattos $x \quad x \quad x$

Handroanthus ochraceus (Cham.) Mattos $\quad x \quad x \quad x$

Handroanthus serratifolius (Vahl) S.O.Grose $\quad x \quad x \quad x$

Handroanthus spongiosus (Rizzini) S.O.Grose $\quad x \quad x$

Continua...

To be continued... 
TABELA 1 Continuação...

TABLE 1 Continued...

\begin{tabular}{l} 
Família/Espécie \\
\hline Jacaranda brasiliana (Lam.) Pers. \\
Jacaranda cuspidifolia Mart. ex A.DC. \\
Tabebuia aurea (Manso) Benth. \& Hook.f. ex \\
S.Moore \\
Tabebuia reticulata A.H. Gentry \\
Tabebuia roseoalba (Ridl.) Sandwith \\
Zeyheria tuberculosa (Vell.) Bureau
\end{tabular}

Bixaceae

Cochlospermum vitifolium (Willd.) Spreng.

Boraginaceae

Cordia glabrata (Mart.) A.DC

Cordia incognita Gottschling \& J.J.Mill.

Cordia sellowiana Cham.

Cordia trichotoma (Vell.) Arrab. ex Steud.

Patagonula bahiensis Moric.

Burseraceae

Commiphora leptophloeos (Mart.) J.B. Gillett

Cactaceae

Cereus jamacaru DC.

Pereskia bahiensis Gürke

Pilosocereus gounellei (F.A.C.Weber) Byles \& G.D.Rowley

Quiabentia zehntneri (Britton \& Rose) Britton \& Rose

Cannabaceae

Celtis brasiliensis (Gardn.) Planch.

Capparaceae

Capparis flexuosa (L.) L.

Caricaceae

Carica sp nova

Celastraceae

Cheiloclinium cognatum (Miers.) A.C.Sm.

Fraunhofera multiflora Mart.

Maytenus quadrangulata (Schrad.) Loes.

Maytenus rigida Mart.

Maytenus robusta Reissek

Maytenus salicifolia Reissek

Salacia crassifolia (Mart. ex Schult.) G.Don

Salacia elliptica (Mart. ex Schult.) G.Don

Combretaceae

Combretum duarteanum Cambess.

Combretum leprosum Mart.

Terminalia argentea (Cambess.) Mart.

TABELA 1 Continuação...

TABLE 1 Continued...

\begin{tabular}{|c|c|c|c|}
\hline \multirow{2}{*}{ Família/Espécie } & \multicolumn{3}{|c|}{ Ambiente } \\
\hline & 1 & 2 & 3 \\
\hline Terminalia glabrescens Mart. & & $x$ & \\
\hline Terminalia januariensis DC. & & & $x$ \\
\hline Terminalia phaeocarpa Eichler & $x$ & & \\
\hline \multicolumn{4}{|l|}{ Ebenaceae } \\
\hline Diospyros hispida A.DC. & & & $x$ \\
\hline Diospyros inconstans Jacq. & & $x$ & \\
\hline \multicolumn{4}{|l|}{ Erythroxylaceae } \\
\hline Erythroxylum betulaceum Mart. & $x$ & $x$ & \\
\hline Erythroxylum caatingae Plowman & & $x$ & \\
\hline Erythroxylum deciduum A.St.-Hil. & $x$ & & $x$ \\
\hline Erythroxylum pelleterianum A.St.-Hil. & & & $x$ \\
\hline Erythroxylum revolutum Mart. & $x$ & $x$ & $x$ \\
\hline
\end{tabular}

Euphorbiaceae

$\mathrm{x} \times \mathrm{x}$

$x \quad x$

Actinostemon klotzschii (Didr.) Pax

Alchornea triplinervia (Spreng.) Müll.Arg.

Aparisthmium cordatum (Juss.) Baill.

Cnidoscolus oligandrus (Müll.Arg.) Pax

Jatropha mollissima (Pohl) Baill.

$\mathrm{x} \quad \mathrm{x}$

$\mathrm{x}$

$\mathrm{X} \times \mathrm{X}$

$\mathrm{x} \quad \mathrm{x}$

$x$

$\mathrm{x}$

$\mathrm{x}$

$x$

$\mathrm{x}$

$x \quad x$

$\mathrm{x} \mathrm{x}$

$\mathrm{x}$

$\mathrm{x}$

$x \times$

$x \times$

$\mathrm{x} \times$

Continua...

To be continued...
Manihot anomala Pohl

Manihot caerulescens Pohl

Manihot grahamii Hook.

Sapium argutum (Müll.Arg.) Huber

Sapium glandulosum (L.) Morong

Sapium haematospermum Müll.Arg.

Sebastiania brasiliensis Spreng.

Sebastiania klotzschiana (Müll.Arg.) Müll.Arg.

Fabaceae

Acacia langsdorffii Benth.

Acosmium lentiscifolium Schott

Albizia inundata (Mart.) Barneby \& J.W.Grimes

Albizia niopoides (Spruce) Burkart

Amburana cearensis (Allemão) A.C.Sm

Anadenanthera colubrina (Vell.) Brenan

Anadenanthera peregrina (L.) Speg.

Andira fraxinifolia Benth.

Bauhinia acuruana Moric.

Bauhinia catingae Harms

Bauhinia cheilantha (Bong.) Steud.

Bauhinia forficata Link

Bauhinia holophylla (Bong.) Steud.

Bauhinia longifolia (Bong.) D.Dietr.

Bauhinia rufa (Bong.) Steud.

Continua... To be continued. 
TABELA1 Continuação...

TABLE 1 Continued...

\begin{tabular}{|c|c|c|c|}
\hline \multirow{2}{*}{ Família/Espécie } & \multicolumn{3}{|c|}{ Ambiente } \\
\hline & 1 & 2 & 3 \\
\hline $\begin{array}{l}\text { Blanchetiodendron blanchetii (Benth.) Barneby \& } \\
\text { J.W.Grimes }\end{array}$ & $\mathrm{x}$ & & \\
\hline Calliandra foliolosa Benth. & $x$ & $\mathrm{x}$ & $x$ \\
\hline Cassia ferruginea (Schrad.) Schrad. ex DC. & & $\mathrm{x}$ & $x$ \\
\hline $\begin{array}{l}\text { Centrolobium sclerophyllum H.C.Lima ex } \\
\text { G.P.Lewis }\end{array}$ & & $x$ & \\
\hline Centrolobium tomentosum Guillem. ex Benth. & $x$ & & \\
\hline Chloroleucon dumosum (Benth.) G.P.Lewis & & $x$ & \\
\hline Chloroleucon foliolosum (Benth.) G.P.Lewis & & $\mathrm{x}$ & $x$ \\
\hline Copaifera langsdorffii Desf. & & & $x$ \\
\hline Copaifera martii Hayne & & $x$ & \\
\hline Dalbergia acuta Benth. & & $x$ & $x$ \\
\hline Dalbergia cearensis Ducke & & $\mathrm{x}$ & $x$ \\
\hline Deguelia nitidula (Benth.) Az.-Tozzi & & $x$ & \\
\hline Diplotropis ferruginea Benth. & $x$ & $x$ & \\
\hline Enterolobium contortisiliquum (Vell.) Morong & $x$ & $x$ & $x$ \\
\hline Enterolobium timbouva Mart. & & $x$ & \\
\hline Goniorrhachis marginata Taub. & $x$ & $\mathrm{x}$ & $x$ \\
\hline Holocalyx balansae Micheli & $\mathrm{x}$ & $\mathrm{x}$ & \\
\hline Hymenaea eriogyne Benth. & & $\mathrm{x}$ & \\
\hline Hymenaea martiana Hayne & & $\mathrm{x}$ & \\
\hline Inga thibaudiana DC. & $x$ & & \\
\hline Leucochloron incuriale (Vell.) Barneby \& J.W.Grimes & $x$ & & \\
\hline Leucochloron limae Barneby \& J.W.Grimes & $\mathrm{x}$ & $\mathrm{x}$ & $x$ \\
\hline Libidibia ferrea (Mart. ex Tul.) L.P.Queiroz & & $\mathrm{x}$ & \\
\hline Lonchocarpus campestris Mart. ex Benth. & & $\mathrm{x}$ & \\
\hline $\begin{array}{l}\text { Lonchocarpus montanus A.M.G.Azevedo ex } \\
\text { M.J.Silva \& A.M.G.Azevedo }\end{array}$ & & $x$ & \\
\hline Lonchocarpus sericeus (Poiret) DC. & $x$ & $x$ & $x$ \\
\hline Luetzelburgia andradelimae H.C.Lima & $x$ & $\mathrm{x}$ & \\
\hline Luetzelburgia auriculata (Allemão) Ducke & & $x$ & \\
\hline Machaerium acutifolium Vogel & $x$ & $\mathrm{x}$ & $x$ \\
\hline Machaerium dimorphandrum Hoehne & $\mathrm{x}$ & $x$ & $\mathrm{x}$ \\
\hline Machaerium floridum (Mart. ex Benth.) Ducke & & $\mathrm{x}$ & \\
\hline Machaerium hirtum (Vell.) Stellfeld & $x$ & $x$ & $x$ \\
\hline Machaerium nictitans (Vell.) Benth. & $x$ & & \\
\hline Machaerium punctatum (Poir.) Pers. & & $x$ & $x$ \\
\hline Machaerium scleroxylon Tul. & $x$ & $\mathrm{x}$ & $x$ \\
\hline Machaerium stipitatum (DC.) Vogel & $x$ & & \\
\hline Machaerium villosum Vogel & & $\mathrm{x}$ & $x$ \\
\hline Mimosa hexandra Micheli & & $x$ & \\
\hline Mimosa tenuiflora (Willd.) Poir. & & $x$ & \\
\hline Myroxylon peruiferum L.f. & $x$ & & \\
\hline
\end{tabular}

Continua...

To be continued..
TABELA 1 Continuação...

TABLE 1 Continued...

\begin{tabular}{|c|c|c|c|}
\hline \multirow{2}{*}{ Família/Espécie } & \multicolumn{3}{|c|}{ Ambiente } \\
\hline & 1 & 2 & 3 \\
\hline Ormosia arborea (Vell.) Harms & $\mathrm{x}$ & & \\
\hline Parapiptadenia rigida (Benth.) Brenan & & $x$ & \\
\hline Peltophorum dubium (Spreng.) Taub. & $\mathrm{x}$ & $x$ & $x$ \\
\hline Piptadenia gonoacantha (Mart.) J.F.Macbr. & $x$ & $\mathrm{x}$ & $x$ \\
\hline Piptadenia paniculata Benth. & $x$ & & \\
\hline Piptadenia viridiflora (Kunth) Benth. & $x$ & $x$ & $\mathrm{x}$ \\
\hline Plathymenia reticulata Benth. & & $\mathrm{x}$ & $x$ \\
\hline Platycyamus regnellii Benth. & $x$ & $\mathrm{x}$ & \\
\hline Platymiscium floribundum Vogel & $x$ & $x$ & $x$ \\
\hline Platypodium elegans Vogel & $x$ & $\mathrm{x}$ & $x$ \\
\hline Poincianella pluviosa (DC.) L.P.Queiroz & $x$ & $x$ & $x$ \\
\hline Pterocarpus zehntneri Harms & & $\mathrm{x}$ & \\
\hline Pterogyne nitens Tul. & $x$ & $\mathrm{x}$ & \\
\hline Senegalia bahiensis (Benth.) Seigler \& Ebinger & $x$ & & \\
\hline Senegalia martii (Benth.) Seigler \& Ebinger & & $x$ & \\
\hline Senegalia polyphylla (DC.) Britton \& Rose & $x$ & $x$ & $x$ \\
\hline $\begin{array}{l}\text { Senna macranthera (Collad.) H.S.Irwin \& } \\
\text { Barneby }\end{array}$ & & $\mathrm{x}$ & \\
\hline Senna spectabilis (DC.) H.S.Irwin \& Barneby & $x$ & $\mathrm{x}$ & $x$ \\
\hline Sloanea stipitata Spruce ex Benth. & $x$ & & \\
\hline Swartzia flaemingii Vogel & & $\mathrm{x}$ & \\
\hline Swartzia myrtifolia J.E.Sm. & & $\mathrm{x}$ & $\mathrm{x}$ \\
\hline Sweetia fruticosa Spreng. & $x$ & $\mathrm{x}$ & $\mathrm{x}$ \\
\hline Vachellia farnesiana (L.) Wight \& Arn. & & $\mathrm{x}$ & \\
\hline Vatairea macrocarpa (Benth.) Ducke & & & $x$ \\
\hline Zollernia ilicifolia (Brongn.) Vogel & $x$ & & $x$ \\
\hline \multicolumn{4}{|l|}{ Lamiaceae } \\
\hline Aegiphila sellowiana Cham. & $x$ & & \\
\hline Vitex laciniosa Turcz. & & $x$ & \\
\hline Vitex megapotamica (Spreng.) Moldenke & & $\mathrm{x}$ & $\mathrm{x}$ \\
\hline \multicolumn{4}{|l|}{ Lauraceae } \\
\hline Nectandra megapotamica (Spreng.) Mez & $x$ & & \\
\hline Nectandra oppositifolia Nees & $\mathrm{x}$ & $\mathrm{x}$ & \\
\hline Ocotea odorifera (Vell.) Rohwer & $x$ & & \\
\hline \multicolumn{4}{|l|}{ Loganiaceae } \\
\hline Strychnos brasiliensis (Spreng.) Mart. & & $\mathrm{x}$ & \\
\hline \multicolumn{4}{|l|}{ Malpighiaceae } \\
\hline Byrsonima crassa Nied. & & & $\mathrm{x}$ \\
\hline Heteropterys byrsonimifolia A.Juss. & & & $x$ \\
\hline Ptilochaeta bahiensis Turcz. & $x$ & $\mathrm{x}$ & $\mathrm{x}$ \\
\hline \multicolumn{4}{|l|}{ Malvaceae } \\
\hline Cavanillesia arborea K. Schum. & $\mathrm{x}$ & $x$ & $x$ \\
\hline Cavanillesia umbellata Ruiz \& Pav. & & $x$ & \\
\hline
\end{tabular}

Continua... To be continued... 
TABELA 1 Continuação...

TABLE 1 Continued...

\begin{tabular}{|c|c|c|c|}
\hline \multirow{2}{*}{ Família/Espécie } & \multicolumn{3}{|c|}{ Ambiente } \\
\hline & 1 & 2 & 3 \\
\hline Ceiba pubiflora (A.St.-Hil.) K.Schum. & $\mathrm{x}$ & $\mathrm{x}$ & $\mathrm{x}$ \\
\hline Ceiba speciosa (A.St.-Hil.) Ravenna & $\mathrm{x}$ & & \\
\hline Ceiba tomentosa(B.L.Rob.) Britten\&Baker F. & & $x$ & \\
\hline Guazuma ulmifolia Lam. & $x$ & $x$ & $\mathrm{x}$ \\
\hline Luehea candicans Mart. \& Zucc. & & $\mathrm{x}$ & $x$ \\
\hline Luehea divaricata Mart. & $\mathrm{x}$ & $x$ & $\mathrm{x}$ \\
\hline Luehea grandiflora Mart. \& Zucc. & & $x$ & \\
\hline Luehea paniculata Mart. & & $\mathrm{x}$ & \\
\hline $\begin{array}{l}\text { Pseudobombax longiflorum (Mart. \& Zucc.) } \\
\text { A.Robyns }\end{array}$ & & $\mathrm{x}$ & $\mathrm{x}$ \\
\hline Pseudobombax marginatum (A.St.-Hil.) A.Robyns & & $\mathrm{x}$ & $\mathrm{x}$ \\
\hline $\begin{array}{l}\text { Pseudobombax tomentosum (Mart. \& Zucc.) } \\
\text { A.Robyns }\end{array}$ & & & $\mathrm{x}$ \\
\hline Sterculia excelsa Mart. & & $x$ & \\
\hline Sterculia striata A.St.-Hill. \& Naudin & $\mathrm{x}$ & $\mathrm{x}$ & $\mathrm{x}$ \\
\hline \multicolumn{4}{|l|}{ Meliaceae } \\
\hline Cabralea canjerana (Vell.) Mart. & & $x$ & \\
\hline Cedrela fissilis Vell. & $x$ & $x$ & $x$ \\
\hline Guarea kunthiana A.Juss. & $x$ & $x$ & \\
\hline Guarea macrophylla Vahl & $x$ & & \\
\hline Trichilia casaretti C.DC. & $x$ & $\mathrm{x}$ & $\mathrm{x}$ \\
\hline Trichilia catigua A.Juss. & $x$ & $\mathrm{x}$ & $\mathrm{x}$ \\
\hline Trichilia hirta L. & $x$ & $\mathrm{x}$ & $\mathrm{x}$ \\
\hline Trichilia pallens C.DC. & $x$ & & $\mathrm{x}$ \\
\hline Trichilia pallida Sw. & & & $x$ \\
\hline \multicolumn{4}{|l|}{ Moraceae } \\
\hline Brosimum gaudichaudii Trécul & & $x$ & \\
\hline Ficus adhatodifolia Schott ex Spreng. & & $x$ & \\
\hline Ficus bonijesulapensis R.M.Castro & $x$ & $x$ & \\
\hline Ficus gomelleira Kunth \& Bouché & $\mathrm{x}$ & $x$ & \\
\hline Ficus pertusa L.f. & $x$ & & \\
\hline Ficus rupicola C.C.Berg \& Carauta & & $x$ & \\
\hline Maclura tinctoria (L.) Steud. & $x$ & $\mathrm{x}$ & $\mathrm{x}$ \\
\hline \multicolumn{4}{|l|}{ Myrsinaceae } \\
\hline Cybianthus amplus (Mez) G.Agostini & $x$ & & \\
\hline Cyclolobium brasiliense Benth. & & & $x$ \\
\hline \multicolumn{4}{|l|}{ Myrtaceae } \\
\hline Blepharocalyx salicifolius (Kunth) O.Berg & $x$ & & $\mathrm{x}$ \\
\hline Calyptranthes widgreniana O.Berg & $x$ & & \\
\hline Campomanesia guazumifolia (Cambess.) O.Berg & & $\mathrm{x}$ & $\mathrm{x}$ \\
\hline Campomanesia velutina (Cambess.) O.Berg & & $x$ & \\
\hline Campomanesia xanthocarpa (Mart.) O.Berg & $x$ & & \\
\hline Eugenia dysenterica DC. & & & $\mathrm{x}$ \\
\hline
\end{tabular}

Continua...

To be continued...
TABELA 1 Continuação...

TABLE 1 Continued...

\begin{tabular}{|c|c|c|c|}
\hline \multirow{2}{*}{ Família/Espécie } & \multicolumn{3}{|c|}{ Ambiente } \\
\hline & 1 & 2 & 3 \\
\hline Eugenia florida DC. & & $x$ & $\mathrm{x}$ \\
\hline Eugenia hyemalis Cambess. & $x$ & & \\
\hline Eugenia ligustrina (Sw.) Willd. & & $x$ & $x$ \\
\hline Eugenia sonderiana O.Berg & & & $x$ \\
\hline Eugenia uniflora L. & $x$ & $x$ & \\
\hline Marlierea laevigata (DC.) Kiaersk. & & $x$ & \\
\hline Marlierea racemosa (Vell.) Kiaersk. & $x$ & & \\
\hline Myrcia guianensis (Aubl.) DC. & & & $x$ \\
\hline Myrcia splendens (Sw.) DC. & & & $x$ \\
\hline Myrcia tomentosa (Aubl.) DC. & & & $x$ \\
\hline Myrciaria floribunda (H.West ex Willd.) O.Berg & $x$ & $\mathrm{x}$ & \\
\hline Myrciaria tenella (DC.) O.Berg & $x$ & & $x$ \\
\hline Plinia cauliflora (Mart.) Kausel & $x$ & & \\
\hline Psidium guajava L. & & $\mathrm{x}$ & \\
\hline Psidium salutare (Kunth) O.Berg & & $x$ & $x$ \\
\hline Siphoneugena densiflora O.Berg & $\mathrm{x}$ & $\mathrm{x}$ & \\
\hline Siphoneugena reitzii D.Legrand & $x$ & & \\
\hline \multicolumn{4}{|l|}{ Nyctaginaceae } \\
\hline Bougainvillea stipitata Griseb. & & $x$ & $x$ \\
\hline Guapira graciliflora (Schmidt) Lundell & $x$ & & $x$ \\
\hline Guapira opposita (Vell.) Reitz & $x$ & $x$ & $x$ \\
\hline Guapira venosa (Choisy) Lundell & $x$ & $x$ & $x$ \\
\hline \multicolumn{4}{|l|}{ Olacaceae } \\
\hline Ximenia americana L. & & $x$ & \\
\hline Ximenia coriacea Engl. & $\mathrm{x}$ & & \\
\hline \multicolumn{4}{|l|}{ Opiliaceae } \\
\hline Agonandra excelsa Griseb. & & $x$ & $x$ \\
\hline \multicolumn{4}{|l|}{ Phyllanthaceae } \\
\hline Margaritaria nobilis L.f. & & $x$ & $x$ \\
\hline Phyllanthus acuminatus Vahl & $x$ & & \\
\hline \multicolumn{4}{|l|}{ Phytolaccaceae } \\
\hline Seguieria langsdorffii Moq. & $x$ & & \\
\hline \multicolumn{4}{|l|}{ Piperaceae } \\
\hline Piper aduncum L. & $x$ & & \\
\hline Piper gaudichaudianum Kunth & & & $x$ \\
\hline \multicolumn{4}{|l|}{ Polygonaceae } \\
\hline Coccoloba alnifolia Casar. & & $x$ & \\
\hline Coccoloba declinata (Vell.) Mart. & $x$ & & \\
\hline Coccoloba schwackeana Lindau & $\mathrm{x}$ & $x$ & $\mathrm{x}$ \\
\hline Ruprechtia apetala Weddell & $x$ & $x$ & \\
\hline Ruprechtia fagifolia Meisn. & $x$ & $x$ & \\
\hline Ruprechtia laxiflora Meisn. & $x$ & $x$ & $x$ \\
\hline
\end{tabular}

To be continued... 
TABELA 1 Continuação...

TABLE 1 Continued...

\begin{tabular}{|c|c|c|c|}
\hline \multirow{2}{*}{ Família/Espécie } & \multicolumn{3}{|c|}{ Ambiente } \\
\hline & 1 & 2 & 3 \\
\hline Triplaris gardneriana Weddell & $\mathrm{x}$ & $x$ & $x$ \\
\hline \multicolumn{4}{|l|}{ Proteaceae } \\
\hline Roupala montana Aubl. & & & $x$ \\
\hline \multicolumn{4}{|l|}{ Rhamnaceae } \\
\hline Rhamnidium elaeocarpum Reissek & & $x$ & $x$ \\
\hline Ziziphus joazeiro Mart. & & $x$ & \\
\hline \multicolumn{4}{|l|}{ Rubiaceae } \\
\hline Alseis floribunda Schott & $x$ & $x$ & \\
\hline Alseis pickelii Pilger \& Schmale & $x$ & $\mathrm{x}$ & \\
\hline Chomelia sericea Müll. Arg. & $x$ & & $x$ \\
\hline Cordiera concolor (Cham.) Kuntze & & $\mathrm{x}$ & $x$ \\
\hline Cordiera elliptica Kuntze & & $\mathrm{x}$ & \\
\hline Cordiera sessilis (Vell.) Kuntze & & & $x$ \\
\hline Coutarea hexandra (Jacq.) K.Schum. & & & $x$ \\
\hline Genipa americana L. & & $x$ & \\
\hline Guettarda angelica Mart. ex Müll.Arg. & & $x$ & \\
\hline Guettarda pohliana Müll.Arg. & $x$ & $x$ & $x$ \\
\hline Guettarda viburnoides Cham. \& Schltdl. & & & $x$ \\
\hline Ixora brevifolia Benth. & $x$ & & \\
\hline $\begin{array}{l}\text { Machaonia brasiliensis (Hoffmanss. ex Humb.) } \\
\text { Cham. \& Schltdl. }\end{array}$ & $\mathrm{x}$ & $\mathrm{x}$ & $\mathrm{x}$ \\
\hline Randia armata (Sw.) DC. & $\mathrm{x}$ & $\mathrm{x}$ & $x$ \\
\hline Randia nitida (Kunth) DC. & $\mathrm{x}$ & $x$ & $x$ \\
\hline Tocoyena formosa (Cham. \& Schltdl.) K.Schum. & $\mathrm{x}$ & & $x$ \\
\hline \multicolumn{4}{|l|}{ Rutaceae } \\
\hline Conchocarpus pentandrus (Engl.) Kallunki \& Pirani & $i x$ & & \\
\hline Galipea ciliata Taub. & & $\mathrm{x}$ & \\
\hline Pilocarpus pennatifolius Lem. & & & $x$ \\
\hline Zanthoxylum caribaeum Lam. & $\mathrm{x}$ & $x$ & $x$ \\
\hline Zanthoxylum fagara (L.) Sargent & $x$ & & $x$ \\
\hline Zanthoxylum petiolare A.St.-Hil. \& Tul. & & & $x$ \\
\hline Zanthoxylum rhoifolium Lam. & $\mathrm{x}$ & & \\
\hline Zanthoxylum riedelianum Engl. & $\mathrm{x}$ & $x$ & \\
\hline Zanthoxylum stelligerum Turcz. & & $\mathrm{x}$ & \\
\hline \multicolumn{4}{|l|}{ Salicaceae } \\
\hline Casearia decandra Jacq. & $x$ & & \\
\hline Casearia rupestris Eichler & & & $x$ \\
\hline Casearia selloana Eichl. & $x$ & $x$ & \\
\hline Prockia crucis P.Browne ex L. & $x$ & & \\
\hline \multicolumn{4}{|l|}{ Sapindaceae } \\
\hline $\begin{array}{l}\text { Allophylus edulis (A.St.-Hil., Cambess. \& A.Juss.) } \\
\text { Radlk. }\end{array}$ & $\mathrm{x}$ & $x$ & \\
\hline
\end{tabular}

Continua...

To be continued...
TABELA 1 Continuação...

TABLE 1 Continued...

\begin{tabular}{|c|c|c|c|}
\hline \multirow{2}{*}{ Família/Espécie } & \multicolumn{3}{|c|}{ Ambiente } \\
\hline & 1 & 2 & 3 \\
\hline Allophylus racemosus Sw. & $\mathrm{x}$ & $\mathrm{x}$ & $\mathrm{x}$ \\
\hline Cupania vernalis Cambess. & & & $x$ \\
\hline Dilodendron bipinnatum Radlk. & & $x$ & $x$ \\
\hline Magonia pubescens A.St.-Hil. & & $x$ & $x$ \\
\hline Talisia esculenta (A.St.-Hil.) Radlk. & & $x$ & $x$ \\
\hline \multicolumn{4}{|l|}{ Sapotaceae } \\
\hline $\begin{array}{l}\text { Chrysophyllum gonocarpum (Mart. \& Eichler) } \\
\text { Engl. }\end{array}$ & $\mathrm{x}$ & & \\
\hline Chrysophyllum marginatum (Hook. \& Arn.) Radlk. & $\mathrm{x}$ & & \\
\hline Micropholis venulosa (Mart. \& Eichler) Pierre & $\mathrm{x}$ & & \\
\hline Pouteria gardneri (Mart. \& Miq.) Baehni & $\mathrm{x}$ & $\mathrm{x}$ & $\mathrm{x}$ \\
\hline \multicolumn{4}{|l|}{ Solanaceae } \\
\hline Capsicum parviflorum Sendtn. & $\mathrm{x}$ & & \\
\hline \multicolumn{4}{|l|}{ Thymelaeaceae } \\
\hline Daphnopsis fasciculata (Meisn.) Nevling & $\mathrm{x}$ & & \\
\hline \multicolumn{4}{|l|}{ Urticaceae } \\
\hline Cecropia glaziovii Snethl. & $\mathrm{x}$ & & \\
\hline Cecropia pachystachya Trécul & $\mathrm{x}$ & $\mathrm{x}$ & \\
\hline Cecropia saxatilis Snethl. & & $x$ & \\
\hline Hemistylus brasiliensis Wedd. Ex Warm. & $\mathrm{x}$ & & \\
\hline Urera baccifera (L.) Gaudich. ex Wedd. & $\mathrm{x}$ & $\mathrm{x}$ & $\mathrm{x}$ \\
\hline \multicolumn{4}{|l|}{ Verbenaceae } \\
\hline Aloysia virgata (Ruiz \& Pav.) A.Juss. & $x$ & $x$ & $x$ \\
\hline Lantana fulcata Lindl. & & $x$ & \\
\hline \multicolumn{4}{|l|}{ Vochysiaceae } \\
\hline Callisthene fasciculata (Spreng.) Mart. & & $x$ & $\mathrm{x}$ \\
\hline Qualea cordata (Mart.) Spreng. & & & $x$ \\
\hline
\end{tabular}

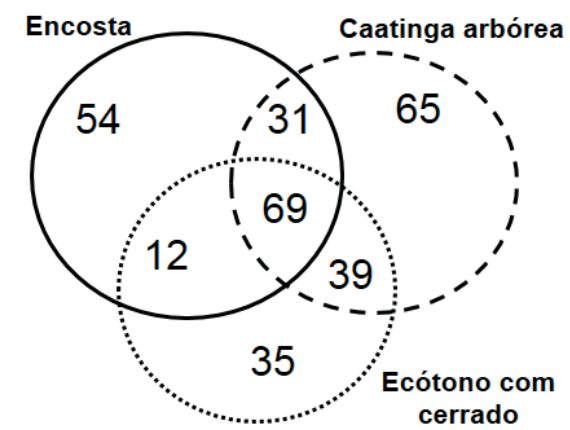

FIGURA 1 Diagrama de Venn produzido a partir do número de espécies compartilhadas e exclusivas entre os ambientes (Encosta, Caatinga arbórea e Ecótono com cerrado) no Parque Nacional Cavernas do Peruaçu.

FIGURE 1 Venn Diagrams resulted from the number of shared and exclusive species among the environments (Slope, Arboreal Caatinga and Savanna Ecotone) in Cavernas do Peruaçu National Park. 
Muitas das espécies exclusivas de cada ambiente são consideradas típicas de determinado ambiente. Como Senegalia bahiensis, Zanthoxylum rhoifolium, espécies exclusivas da encosta mais úmida, são consideradas como típicas do domínio atlântico, segundo Santos et al. (20l2). Ainda de acordo com esses mesmos autores Casearia rupestris, Diospyros hispida, Pseudobombax tomentosum, Callisthene fasciculata, Dilodendron bipinnatum, Jacaranda brasiliana, Magonia pubescens, Terminalia argentea são características de enclaves de florestas decíduas em matriz de cerrado. As três primeiras apresentaram ocorrência restrita na área ecotonal com cerrado, as demais também ocorreram na Caatinga arbórea. Já, Cavanillesia umbellata, Centrolobium sclerophyllum, Lonchocarpus montanus, Vitex laciniosa, Zanthoxylum stelligerum foram exclusivas da Caatinga arbórea, as quais são características desse ambiente. Chloroleucon dumosu, Cochlospermum vitifolium, Pilosocereus gounellei, Ziziphus joazeiro, Galipea ciliata e Pterocarpus zehntneri também foram exclusivas da Caatinga arbórea, sendo as quatro primeiras características da caatinga do cristalino e as duas últimas típicas de afloramento. Esse resultado demonstra o complexo vegetacional que é a região do presente estudo, onde um grande número de espécies é restrito a determinadas características microambientais na região (SANTOS et al., 20I2). Esse fato justifica a conservação imediata dessas áreas.

\section{Estrutura}

Foram amostrados 1391 indivíduos, resultando em uma estimativa de 1375 indivíduos·ha-1. Já, nos três ambientes separadamente, encosta, Caatinga arbórea e ecótono foram amostrados 429, 706 e 256 indivíduos, respectivamente, correspondendo a 1340,62; 1765 e 1280 indivíduos $\cdot \mathrm{ha}^{-1}$, respectivamente. Esse resultado está dentro dos padrões encontrados por Rodal et al. (2008), em caatingas arbóreas e arbustivas ( 1076 a 2172 ind $\left.\cdot h^{-1}\right)$. $O$ valor encontrado para a área de Caatinga arbórea está próximo ao encontrado em caatingas arbóreas no norte de Minas por Santos et al. (201 I) (I652,5 ind $\cdot$ ha $\left.^{-1}\right)$.

O índice de diversidade de Shannon e a equabilidade de Pielou foram 4, 19 nats·indivíduo-1 e 0,84, respectivamente. Já, para as áreas separadamente, o índice de diversidade encontrado foi 3,49, 3,74 e 3,6I nats.indivíduo-1 ${ }^{-1}$ para a área de encosta, Caatinga arbórea e área ecotonal, respectivamente. $A$ equabilidade foi de $0,80,0,84$ e 0,85, respectivamente. A diversidade para os três ambientes foi superior ao padrão encontrado para áreas deciduais, como (SANTOS et al., 201 I) que obteve uma diversidade de 2,94, em uma área de Caatinga arbórea no norte de Minas, Alcoforado-Filho et al. (2003) (3,09 nats·indivíduo $\left.{ }^{-1}\right)$ em uma vegetação Caducifólia Espinhosa em Caruaru, Pernambuco e Silva e Scariot (2004) (3, 18 nats·indivíduo-1) em uma Floresta Estacional Decidual em Afloramento Calcário na Bacia do Rio Paranã, GO.

A área basal encontrada foi de $31,92 \mathrm{~m}^{2}$, o que corresponde a $34,70 \mathrm{~m}^{2} \cdot \mathrm{ha}^{-1}$. Entre os ambientes foi I 5,25 $\mathrm{m}^{2}$ na área de encosta, 12,02 $\mathrm{m}^{2}$ na Caatinga arbórea e $4,65 \mathrm{~m}^{2}$ no ecótono com cerrado. O equivalente à $47,64,30,05$ e $23,29 \mathrm{~m}^{2} \cdot \mathrm{ha}^{-1}$, respectivamente. 0 que está acima da encontrada por Rodal et al. (2008) ( I4,62 a 34,29 $\left.\mathrm{m}^{2} \cdot \mathrm{ha}^{-1}\right)$ e por Santos et al. (20I I) (16, I0 $\left.\mathrm{m}^{2} \cdot \mathrm{ha}^{-1}\right)$, Alcoforado-Filho et al. (2003) $\left(24,9 \mathrm{~m}^{2} \cdot \mathrm{ha}^{-1}\right)$ e Silva e Scariot (2004) (18,63 $\left.\mathrm{m}^{2} \cdot \mathrm{ha}^{-1}\right)$. A área de encosta apresentou menor número de indivíduos e maior valor de área basal. Já, a Caatinga arbórea teve comportamento contrário, apresentando maior densidade e menor área basal. Demonstrando que algumas fisionomias investem mais no crescimento em biomassa, como a área mais úmida. Enquanto que outras, como a Caatinga arbórea para compensar o pouco investimento em área basal investem na reprodução, apresentando maior quantidade de indivíduos, ou seja, maior densidade. É provável que parte dessa variação quanto à densidade, esteja ligada à disponibilidade hídrica, como a distribuição da chuva ao longo do ano e a retenção de água no solo (ALCOFARADO FILHO et al., 2003).

Essas áreas também respondem estruturalmente de forma diferente quanto às espécies dominantes (Tabela 2). Ficus bonijesulapensis, Handroanthus ochraceus, Goniorrhachis marginata, Casearia selloana e Bauhinia forficata representaram $37,42 \%$ do valor de importância para a área de encosta. Esses altos valores de importância devemse a alta densidade de $H$. ochraceus, a alta dominância de $F$. bonijesulapensis e alta frequência das demais. Cavanillesia arborea, Myracrodruon urundeuva, Handroanthus ochraceus, Poincianella pluviosa e Galipea ciliata representaram $29,39 \%$ do $\mathrm{VI}$ total para a área de Caatinga arbórea. $\bigcirc$ que está relacionado à alta densidade de P.pluviosa e G. ciliata, contudo em extratos diferentes, sendo a primeira nas maiores classes de altura e a segunda nas menores. Já, o alto VI de C. arborea está relacionado ao grande porte dos indivíduos refletindo na alta dominância, enquanto que as demais espécies obtiveram alta frequência. No ambiente de ecótono Aspidosperma multiflorum, Myracrodruon urundeuva, 
Callisthene fasciculata, Handroanthus impetiginosus e Pseudobombax marginatum representaram 44,7I\% do VI total. Esses valores de importância podem ser atribuídos à alta densidade, dominância e frequência de A. multiflorum, a alta frequência de $M$. urundeuva e a alta dominância de $C$. fasciculata, P. marginatum, e $\mathrm{H}$. impetiginosus.

TABELA2 Espécies amostradas no levantamento estrutural, com seus respectivos parâmetros fitossociológicos em um remanescente de Floresta Tropical Sazonalmente Seca no Parque Nacional Cavernas do Peruaçu, MG, DA - Densidade Absoluta (ind,ha-1); DoA - Dominância Absoluta $\left(\mathrm{m}^{2}\right.$,ha-1); FA - Frequência Absoluta (\%) e VI = Valor de Importância (\%),

TABLE 2 Species surveyed in Cavernas do Peruaçu National Park, followed by the phytosociological parameters: DA - Absolute Density (ind,ha-1); DoA - Absolute Dominance $\left(\mathrm{m}^{2}\right.$,ha-1 $)$; FA - Absolute Frequency $(\%)$ and VI = Importance Value (\%),

\begin{tabular}{|c|c|c|c|c|c|c|c|c|c|c|c|c|}
\hline \multirow{3}{*}{ Espécie } & \multicolumn{12}{|c|}{ Ambiente } \\
\hline & \multicolumn{4}{|c|}{ Encosta } & \multicolumn{4}{|c|}{ Caatinga arbórea } & \multicolumn{4}{|c|}{ Ecótono } \\
\hline & DA & DoA & FA & VI & DA & DoA & FA & $\mathrm{VI}$ & DA & DoA & FA & VI \\
\hline Acosmium lentiscifolium & 6,25 & 0,13 & 25,0 & 0,66 & & & & & & & & \\
\hline Allophylus edulis & 12,50 & 0,03 & 25,0 & 0,75 & & & & & & & & \\
\hline Allophylus racemosus & 6,25 & 0,02 & 25,0 & 0,59 & & & & & & & & \\
\hline Aloysia virgata & & & & & 2,50 & 0,00 & 10 & 0,20 & & & & \\
\hline Alseis pickelii & 15,63 & 0,39 & 25,0 & 1,08 & 2,50 & 0,00 & 10 & 0,20 & & & & \\
\hline Anadenanthera colubrina & 3,13 & 0,04 & 12,5 & 0,32 & 70,00 & 0,78 & 70 & 3,27 & & & & \\
\hline Annona leptopetala & & & & & 7,50 & 0,01 & 20 & 0,46 & 10,0 & 0,01 & 20 & 0,58 \\
\hline Annona vepretorum & & & & & 10,00 & 0,05 & 10 & 0,40 & & & & \\
\hline Aralia excelsa & & & & & 7,50 & 0,08 & 20 & 0,54 & 5,0 & 0,03 & 20 & 0,48 \\
\hline Arrabidaea bahiensis & & & & & 105 & 0,39 & 30 & 2,87 & & & & \\
\hline Aspidosperma cuspa & 9,38 & 0,16 & 12,5 & 0,55 & 17,50 & 0,21 & 50 & 1,33 & 20,0 & 0,39 & 40 & 1,69 \\
\hline Aspidosperma cylindrocarpon & & & & & 5,00 & 0,01 & 20 & 0,41 & & & & \\
\hline Aspidosperma multiflorum & & & & & 12,50 & 0,17 & 20 & 0,73 & 205 & 4,40 & 100 & 13,16 \\
\hline Aspidosperma polyneuron & 9,38 & 0,09 & 25,0 & 0,71 & & & & & & & & \\
\hline Aspidosperma pyrifolium & 6,25 & 0,03 & 25,0 & 0,59 & 25,00 & 0,15 & 50 & 1,42 & 15,0 & 0,02 & 40 & 1,03 \\
\hline Aspidosperma subincanum & 40,63 & 0,84 & 37,5 & 2,22 & & & & & & & & \\
\hline Astronium fraxinifolium & 3,13 & 0,02 & 12,5 & 0,30 & & & & & 35,0 & 0,51 & 60 & 2,55 \\
\hline Bauhinia acuruana & & & & & 7,50 & 0,02 & 20 & 0,47 & 20,0 & 0,04 & 40 & 1,19 \\
\hline Bauhinia catingae & & & & & 7,50 & 0,02 & 20 & 0,47 & 5,0 & 0,05 & 20 & 0,51 \\
\hline Bauhinia cheilantha & 3,13 & 0,01 & 12,5 & 0,29 & & & & & 5,0 & 0,01 & 20 & 0,45 \\
\hline Bauhinia forficata & 56,25 & 0,14 & 62,5 & 2,53 & & & & & & & & \\
\hline Bauhinia rufa & & & & & 52,50 & 0,17 & 40 & 1,80 & 10,0 & 0,03 & 40 & 0,92 \\
\hline Blanchetiodendron blanchetii & 3,13 & 0,02 & 12,5 & 0,30 & & & & & & & & \\
\hline Brosimum gaudichaudii & & & & & 5,00 & 0,01 & 10 & 0,26 & & & & \\
\hline Calliandra foliolosa & 9,38 & 0,03 & 37,5 & 0,87 & 10,00 & 0,04 & 10 & 0,39 & & & & \\
\hline Callisthene fasciculata & & & & & & & & & 60,0 & 2,62 & 60 & 6,23 \\
\hline Campomanesia velutina & & & & & 7,50 & 0,03 & 20 & 0,49 & & & & \\
\hline Campomanesia xanthocarpa & 3,13 & 0,01 & 12,5 & 0,29 & & & & & & & & \\
\hline Capparis flexuosa & 6,25 & 0,01 & 25,0 & 0,58 & & & & & & & & \\
\hline Capsicum parviflorum & 3,13 & 0,01 & 12,5 & 0,29 & & & & & & & & \\
\hline Casearia selloana & 81,25 & 0,33 & 75,0 & 3,49 & & & & & & & & \\
\hline Cassia ferruginea & & & & & & & & & 20,0 & 0,12 & 40 & 1,30 \\
\hline Cavanillesia arborea & 6,25 & 2,20 & 25,0 & 2,11 & 5,00 & 7,45 & 20 & 8,67 & & & & \\
\hline Cedrela fissilis & 6,25 & 0,05 & 25,0 & 0,60 & 2,50 & 0,01 & 10 & 0,21 & & & & \\
\hline
\end{tabular}


TABELA 2 Continuação...

TABLE 2 Continued...

\begin{tabular}{|c|c|c|c|c|c|c|c|c|c|c|c|c|}
\hline \multirow{3}{*}{ Espécie } & \multicolumn{12}{|c|}{ Ambiente } \\
\hline & \multicolumn{4}{|c|}{ Encosta } & \multicolumn{4}{|c|}{ Caatinga arbórea } & \multicolumn{4}{|c|}{ Ecótono } \\
\hline & DA & DoA & FA & VI & DA & DoA & FA & VI & DA & DoA & FA & VI \\
\hline Ceiba pubiflora & 9,38 & 0,77 & 37,5 & 1,39 & 2,50 & 0,04 & 10 & 0,24 & & & & \\
\hline Ceiba tomentosa(B,L,Rob,) & & & & & 5,00 & 0,80 & 10 & 1,14 & & & & \\
\hline Celtis brasiliensis & 3,13 & 0,01 & 12,5 & 0,29 & & & & & & & & \\
\hline Cereus jamacaru & & & & & 7,50 & 0,28 & 20 & 0,77 & & & & \\
\hline Chomelia sericea & & & & & & & & & 10,0 & 0,02 & 20 & 0,60 \\
\hline Cnidoscolus oligandrus & 9,38 & 0,33 & 37,5 & 1,09 & 2,50 & 0,00 & 10 & 0,21 & & & & \\
\hline Coccoloba declinata & 3,13 & 0,01 & 12,5 & 0,29 & & & & & & & & \\
\hline Coccoloba schwackeana & 9,38 & 0,13 & 25,0 & 0,73 & 17,50 & 0,34 & 40 & 1,33 & & & & \\
\hline Combretum duarteanum & 9,38 & 0,02 & 25,0 & 0,66 & 67,50 & 0,77 & 60 & 3,05 & 35,0 & 0,19 & 40 & 1,79 \\
\hline Combretum leprosum & 28,13 & 0,67 & 37,5 & 1,79 & 77,50 & 0,53 & 60 & 2,98 & 5,0 & 0,00 & 20 & 0,44 \\
\hline Commiphora leptophloeos & 9,38 & 1,52 & 25,0 & 1,71 & 32,50 & 1,10 & 40 & 2,45 & & & & \\
\hline Copaifera langsdorffii & & & & & & & & & 5,0 & 0,22 & 20 & 0,75 \\
\hline Copaifera martii & & & & & 2,50 & 0,00 & 10 & 0,21 & & & & \\
\hline Cordia glabrata & & & & & & & & & 5,0 & 0,02 & 20 & 0,46 \\
\hline Cordia trichotoma & & & & & 2,50 & 0,01 & 10 & 0,21 & 5,0 & 0,02 & 20 & 0,46 \\
\hline Cordiera sessilis & & & & & & & & & 5,0 & 0,01 & 20 & 0,45 \\
\hline Cybianthus amplus & 6,25 & 0,01 & 25,0 & 0,58 & & & & & & & & \\
\hline Cyrtocarpa caatingae & 3,13 & 0,02 & 12,5 & 0,30 & 10,00 & 0,12 & 30 & 0,79 & & & & \\
\hline Dalbergia acuta & & & & & 5,00 & 0,01 & 20 & 0,42 & 5,0 & 0,01 & 20 & 0,45 \\
\hline Dalbergia cearensis & & & & & 7,50 & 0,07 & 20 & 0,53 & 35,0 & 0,69 & 40 & 2,51 \\
\hline Dilodendron bipinnatum & & & & & 2,50 & 0,09 & 10 & 0,30 & 35,0 & 0,95 & 80 & 3,49 \\
\hline Diospyros hispida & & & & & & & & & 5,0 & 0,24 & 20 & 0,77 \\
\hline Diplotropis ferruginea & 3,13 & 0,00 & 12,5 & 0,29 & 27,50 & 0,07 & 30 & 1,06 & & & & \\
\hline Erythroxylum betulaceum & 6,25 & 0,01 & 25,0 & 0,57 & 7,50 & 0,04 & 30 & 0,65 & & & & \\
\hline Erythroxylum revolutum & & & & & 2,50 & 0,00 & 10 & 0,21 & & & & \\
\hline Eugenia dysenterica & & & & & & & & & 20,0 & 0,15 & 40 & 1,35 \\
\hline Eugenia florida & & & & & 2,50 & 0,01 & 10 & 0,21 & 10,0 & 0,06 & 20 & 0,65 \\
\hline Eugenia ligustrina & & & & & 50,00 & 0,09 & 30 & 1,51 & 40,0 & 0,15 & 40 & 1,87 \\
\hline Eugenia sonderiana & & & & & & & & & 5,0 & 0,01 & 20 & 0,45 \\
\hline Eugenia uniflora & 12,50 & 0,07 & 12,5 & 0,56 & 25,00 & 0,36 & 40 & 1,48 & & & & \\
\hline Ficus bonijesulapensis & 6,25 & 21,71 & 25,0 & 15,75 & & & & & & & & \\
\hline Fraunhofera multiflora & & & & & 2,50 & 0,08 & 10 & 0,29 & & & & \\
\hline Galipea ciliata & & & & & 192,50 & 0,70 & 20 & 4,72 & & & & \\
\hline Goniorrhachis marginata & 59,38 & 1,85 & 75,0 & 4,01 & 27,50 & 0,77 & 40 & 2,00 & 10,0 & 0,17 & 20 & 0,81 \\
\hline Guapira venosa & 12,50 & 0,10 & 37,5 & 1,00 & 10,00 & 0,05 & 20 & 0,55 & & & & \\
\hline Guazuma ulmifolia & & & & & & & & & 5,0 & 0,06 & 20 & 0,53 \\
\hline Guettarda pohliana & 6,25 & 0,01 & 12,5 & 0,37 & 2,50 & 0,01 & 10 & 0,21 & 10,0 & 0,02 & 40 & 0,90 \\
\hline Handroanthus heptaphyllus & 15,63 & 0,07 & 37,5 & 1,06 & 2,50 & 0,00 & 10 & 0,20 & & & & \\
\hline Handroanthus impetiginosus & 21,88 & 0,32 & 12,5 & 0,97 & 50,00 & 1,74 & 80 & 4,11 & 30,0 & 2,70 & 40 & 5,26 \\
\hline Handroanthus ochraceus & 309,4 & 3,85 & 75,0 & 11,63 & 90,00 & 1,95 & 70 & 4,95 & 20,0 & 0,07 & 20 & 0,92 \\
\hline
\end{tabular}


TABELA 2 Continuação...

TABLE 2 Continued...

\begin{tabular}{|c|c|c|c|c|c|c|c|c|c|c|c|c|}
\hline \multirow{3}{*}{ Espécie } & \multicolumn{12}{|c|}{ Ambiente } \\
\hline & \multicolumn{4}{|c|}{ Encosta } & \multicolumn{4}{|c|}{ Caatinga arbórea } & \multicolumn{4}{|c|}{ Ecótono } \\
\hline & DA & DoA & FA & VI & $\mathrm{DA}$ & DoA & FA & VI & $\mathrm{DA}$ & DoA & FA & VI \\
\hline Handroanthus spongiosus & & & & & 62,50 & 1,11 & 50 & 3,18 & & & & \\
\hline Heteropterys byrsonimifolia & & & & & & & & & 10,0 & 0,01 & 20 & 0,58 \\
\hline Holocalyx balansae & 18,75 & 0,81 & 12,5 & 1,24 & & & & & & & & \\
\hline Inga thibaudiana & 12,50 & 0,99 & 25,0 & 1,42 & & & & & & & & \\
\hline Jacaranda brasiliana & & & & & 2,50 & 0,18 & 10 & 0,40 & 20,0 & 0,97 & 40 & 2,52 \\
\hline Lantana fulcata & & & & & 7,50 & 0,02 & 10 & 0,32 & & & & \\
\hline Leucochloron incuriale & 3,13 & 0,01 & 12,5 & 0,29 & & & & & & & & \\
\hline Leucochloron limae & 28,13 & 0,88 & 37,5 & 1,94 & & & & & & & & \\
\hline Luehea paniculata & & & & & 5,00 & 0,01 & 10 & 0,26 & & & & \\
\hline Luetzelburgia andradelimae & 3,13 & 0,01 & 12,5 & 0,29 & 17,50 & 0,10 & 20 & 0,75 & & & & \\
\hline Machaerium acutifolium & 3,13 & 0,01 & 12,5 & 0,29 & 27,50 & 0,38 & 50 & 1,71 & 10,0 & 0,22 & 40 & 1,19 \\
\hline Machaerium hirtum & & & & & & & & & 5,0 & 0,12 & 20 & 0,60 \\
\hline Machaerium scleroxylon & & & & & 5,00 & 0,09 & 20 & 0,51 & 10,0 & 0,07 & 20 & 0,66 \\
\hline Machaerium villosum & & & & & 5,00 & 0,18 & 20 & 0,61 & 5,0 & 0,01 & 20 & 0,46 \\
\hline Maclura tinctoria & 6,25 & 0,07 & 25,0 & 0,62 & 2,50 & 0,16 & 10 & 0,38 & & & & \\
\hline Magonia pubescens & & & & & 2,50 & 0,01 & 10 & 0,21 & 15,0 & 0,59 & 20 & 1,55 \\
\hline Manihot caerulescens & 6,25 & 0,05 & 25,0 & 0,60 & & & & & 5,0 & 0,01 & 20 & 0,45 \\
\hline Maytenus quadrangulata & 6,25 & 0,01 & 25,0 & 0,58 & & & & & & & & \\
\hline Maytenus robusta & & & & & & & & & 5,0 & 0,01 & 20 & 0,45 \\
\hline Myracrodruon urundeuva & 12,50 & 1,41 & 50,0 & 2,13 & 57,50 & 3,37 & 90 & 6,21 & 90,0 & 1,69 & 100 & 6,29 \\
\hline Myrcia guianensis & & & & & & & & & 15,0 & 0,02 & 20 & 0,72 \\
\hline Myrcia tomentosa & & & & & & & & & 5,0 & 0,00 & 20 & 0,44 \\
\hline Myrciaria tenella & & & & & & & & & 5,0 & 0,00 & 20 & 0,44 \\
\hline Pereskia bahiensis & & & & & 2,50 & 0,01 & 10 & 0,21 & & & & \\
\hline Piptadenia gonoacantha & & & & & 7,50 & 0,02 & 10 & 0,32 & & & & \\
\hline Piptadenia paniculata & 15,63 & 0,93 & 25,0 & 1,45 & & & & & & & & \\
\hline Piptadenia viridiflora & 3,13 & 0,46 & 12,5 & 0,61 & & & & & 5,0 & 0,01 & 20 & 0,45 \\
\hline Plathymenia reticulata & & & & & 12,50 & 0,27 & 30 & 1,00 & 5,0 & 0,21 & 20 & 0,74 \\
\hline Platypodium elegans & & & & & 22,50 & 0,10 & 20 & 0,84 & 25,0 & 0,27 & 60 & 1,95 \\
\hline Plinia cauliflora & 3,13 & 0,00 & 12,5 & 0,29 & & & & & & & & \\
\hline Poincianella pluviosa & 50,00 & 0,37 & 50,0 & 2,33 & 127,50 & 1,22 & 70 & 4,84 & 90,0 & 0,93 & 40 & 4,29 \\
\hline Pouteria gardneri & & & & & 20,00 & 0,10 & 10 & 0,64 & 5,0 & 0,01 & 20 & 0,46 \\
\hline Prockia crucis & 3,13 & 0,00 & 12,5 & 0,29 & & & & & & & & \\
\hline Pseudobombax longiflorum & & & & & 2,50 & 0,07 & 10 & 0,28 & & & & \\
\hline Pseudobombax marginatum & & & & & 10,00 & 0,05 & 20 & 0,55 & 20,0 & 2,05 & 60 & 4,38 \\
\hline Pterocarpus zehntneri & & & & & 2,50 & 0,23 & 10 & 0,45 & & & & \\
\hline Pterogyne nitens & 9,38 & 1,82 & 37,5 & 2,13 & & & & & & & & \\
\hline Ptilochaeta bahiensis & 9,38 & 0,04 & 25,0 & 0,68 & 35,00 & 0,13 & 40 & 1,43 & & & & \\
\hline Randia armata & 21,88 & 0,08 & 50,0 & 1,43 & 2,50 & 0,01 & 10 & 0,21 & 60,0 & 0,25 & 60 & 2,84 \\
\hline Ruprechtia apetala & 31,25 & 0,88 & 50,0 & 2,22 & 2,50 & 0,02 & 10 & 0,23 & & & & \\
\hline
\end{tabular}


TABELA 2 Continuação...

TABLE 2 Continued...

\begin{tabular}{|c|c|c|c|c|c|c|c|c|c|c|c|c|}
\hline \multirow{3}{*}{ Espécie } & \multicolumn{12}{|c|}{ Ambiente } \\
\hline & \multicolumn{4}{|c|}{ Encosta } & \multicolumn{4}{|c|}{ Caatinga arbórea } & \multicolumn{4}{|c|}{ Ecótono } \\
\hline & DA & DoA & FA & $\mathrm{VI}$ & $\mathrm{DA}$ & DoA & FA & VI & $\mathrm{DA}$ & DoA & FA & $\mathrm{VI}$ \\
\hline Ruprechtia laxiflora & 3,13 & 0,02 & 12,5 & 0,30 & 17,50 & 0,56 & 20 & 1,26 & 5,0 & 0,01 & 20 & 0,45 \\
\hline Salacia crassifolia & & & & & & & & & 5,0 & 0,21 & 20 & 0,73 \\
\hline Sapium argutum & 12,50 & 0,03 & 37,5 & 0,95 & 15,00 & 0,05 & 30 & 0,80 & 10,0 & 0,05 & 20 & 0,63 \\
\hline Schinopsis brasiliensis & 3,13 & 0,04 & 12,5 & 0,32 & 2,50 & 0,29 & 10 & 0,52 & 5,0 & 0,00 & 20 & 0,44 \\
\hline Senegalia bahiensis & 15,63 & 0,07 & 12,5 & 0,64 & & & & & & & & \\
\hline Senegalia martii & & & & & 50,00 & 0,16 & 50 & 1,90 & & & & \\
\hline Senegalia polyphylla & 9,38 & 0,04 & 25,0 & 0,67 & 12,50 & 0,08 & 30 & 0,78 & 25,0 & 0,35 & 40 & 1,76 \\
\hline Senna spectabilis & 3,13 & 0,01 & 12,5 & 0,29 & & & & & & & & \\
\hline Sloanea stipitata & 3,13 & 0,01 & 12,5 & 0,29 & & & & & & & & \\
\hline Spondias mombin & 21,88 & 0,90 & 25,0 & 1,59 & & & & & & & & \\
\hline Spondias tuberosa & 3,13 & 0,04 & 12,5 & 0,31 & & & & & & & & \\
\hline Sterculia excelsa & & & & & 25,00 & 0,60 & 40 & 1,76 & & & & \\
\hline Sterculia striata & 12,50 & 0,25 & 37,5 & 1,11 & & & & & & & & \\
\hline Swartzia flaemingii & & & & & 12,50 & 0,03 & 20 & 0,58 & & & & \\
\hline Sweetia fruticosa & 3,13 & 0,05 & 12,5 & 0,32 & 32,50 & 0,44 & 40 & 1,72 & 10,0 & 0,08 & 20 & 0,68 \\
\hline Syagrus oleracea & & & & & 5,00 & 0,08 & 20 & 0,49 & 15,0 & 0,27 & 40 & 1,39 \\
\hline Tabebuia aurea & & & & & & & & & 5,0 & 0,03 & 20 & 0,48 \\
\hline Tabebuia reticulata & & & & & 7,50 & 0,05 & 30 & 0,66 & 5,0 & 0,15 & 20 & 0,65 \\
\hline Tabebuia roseoalba & 12,50 & 0,04 & 37,5 & 0,96 & 2,50 & 0,01 & 10 & 0,21 & 20,0 & 0,27 & 40 & 1,52 \\
\hline Terminalia argentea & & & & & & & & & 5,0 & 0,09 & 20 & 0,56 \\
\hline Terminalia phaeocarpa & 59,38 & 0,54 & 25,0 & 2,27 & & & & & & & & \\
\hline Tocoyena formosa & & & & & & & & & 20,0 & 0,06 & 60 & 1,52 \\
\hline Trichilia casaretti & 56,25 & 0,43 & 37,5 & 2,32 & 5,00 & 0,01 & 10 & 0,27 & 5,0 & 0,01 & 20 & 0,45 \\
\hline Trichilia catigua & & & & & 12,50 & 0,03 & 10 & 0,43 & & & & \\
\hline Trichilia hirta & 34,38 & 0,29 & 62,5 & 2,09 & 10,00 & 0,09 & 20 & 0,60 & & & & \\
\hline Trichilia pallens & & & & & & & & & 5,0 & 0,09 & 20 & 0,57 \\
\hline Triplaris gardneriana & 3,13 & 0,01 & 12,5 & 0,29 & & & & & & & & \\
\hline Vachellia farnesiana & & & & & 5,00 & 0,01 & 10 & 0,26 & & & & \\
\hline Vitex laciniosa & & & & & 7,50 & 0,01 & 30 & 0,62 & & & & \\
\hline Ximenia americana & & & & & 2,50 & 0,06 & 10 & 0,27 & & & & \\
\hline Ximenia coriacea & 3,13 & 0,00 & 12,5 & 0,29 & & & & & & & & \\
\hline Zanthoxylum petiolare & & & & & & & & & 10,0 & 0,04 & 40 & 0,94 \\
\hline Zollernia ilicifolia & & & & & & & & & 10,0 & 0,13 & 20 & 0,76 \\
\hline
\end{tabular}

Nenhuma espécie esteve entre as cinco mais importantes nas três áreas. Apenas duas espécies foram as mais importantes em duas áreas. Myracrodruon urundeuva na Caatinga arbórea e área ecotonal e Handroanthus ochraceus na encosta e Caatinga arbórea. Myracrodruon urundeuva é uma espécie de ampla distribuição e indicadora de florestas deciduais (PRADO; GIBBS, 1993) e H. ochraceus, segundo Gentry (1992) caracteriza-se como elemento típico do cerrado brasileiro, mas podendo ocorrer também em formações florestais, sendo também típica de Caatinga arbórea (SANTOS et al., 20I2).

Algumas espécies, além de se destacarem quanto ao valor de importância, também se destacaram quanto ao valor de indicação (Tabela 3). Sendo $C$. selloana e B. forficata para a encosta. E A. multiflorum, C. fasciculata e M. urundeuva para o ecótono (Tabela 3). C. selloana é característica de floresta estacional 
semidecidual (MARQUETE; VAZ, 2007), B. forficata é característica da floresta pluvial Atlântica, encontrandose, preferencialmente, em planícies aluviais, úmidas ou início de encostas (LORENZI, 1998) e C. fasciculata é indicadora de enclaves de floresta seca em matriz de cerrado (SANTOS et al., 20I2), confirmando a diferenciação florística e estrutural entre as áreas.

TABELA 3 Espécies indicadoras de três ambientes amostrados no Parque Nacional Cavernas do Peruaçu, Norte de Minas Gerais. VI= valor de indicação

TABLE 3 Indicator species from the three environments sampled in Cavernas do Peruaçu National Park, north of Minas Gerais. VI= Indicator value

\begin{tabular}{|c|c|c|}
\hline \multicolumn{3}{|c|}{ Ecótono com cerrado } \\
\hline Espécie & VI & $p$ \\
\hline Aspidosperma multiflorum & 94,3 & 0,0002 \\
\hline Dilodendron bipinnatum & 74,7 & 0,0012 \\
\hline Callisthene fasciculata & 60 & 0,0044 \\
\hline Tocoyena formosa & 60 & 0,0044 \\
\hline Astronium fraxinifolium & 55,1 & 0,0152 \\
\hline Myracrodruon urundeuva & 56,2 & 0,0154 \\
\hline Cassia ferruginea & 40 & 0,0412 \\
\hline Eugenia dysenterica & 40 & 0,0412 \\
\hline Zanthoxylum petiolare & 40 & 0,0428 \\
\hline \multicolumn{3}{|c|}{ Caatinga arbórea } \\
\hline Espécie & VI & $p$ \\
\hline Anadenanthera colubrina & 67 & 0,0044 \\
\hline Senegalia martii & 50 & 0,0228 \\
\hline Handroanthus spongiosus & 50 & 0,0296 \\
\hline Sterculia excelsa & 40 & 0,0484 \\
\hline \multicolumn{3}{|c|}{ Encosta } \\
\hline Espécie & VI & $p$ \\
\hline Casearia selloana & 75 & 0,0002 \\
\hline Bauhinia forficata & 62,5 & 0,0062 \\
\hline Trichilia hirta & 48,4 & 0,0324 \\
\hline Pterogyne nitens & 37,5 & 0,0356 \\
\hline Sterculia striata & 37,5 & 0,0366 \\
\hline Aspidosperma subincanum & 37,5 & 0,0384 \\
\hline Ruprechtia apetala & 46,3 & 0,0392 \\
\hline
\end{tabular}

Com relação à distribuição dos indivíduos em classes de diâmetro (Figura 2), Caatinga arbórea e encosta apresentaram um mesmo padrão, com uma diminuição gradual do número de indivíduos. Já, o ambiente ecotonal apresentou uma distribuição tendendo a normal, com uma maior concentração de indivíduos nas classes intermediárias. Considerando que a distribuição diamétrica pode refletir ○ histórico da floresta, bem como a ocorrência de distúrbios passados como fogo, corte seletivo e herbivoria (MEYER et al., 196I), o padrão encontrado para a Caatinga arbórea e para a encosta foi diferente do encontrado para outras florestas deciduais, como o trabalho de Santos et al. (20II) amostrando uma floresta decidual no município de Juvenília, norte de Minas. Neste estudo, foi encontrada uma distribuição normal, o que segundo os autores, pode estar relacionado a fatores antrópicos que podem estar interferindo na regeneração natural da área (SANTOS; VIEIRA, 2005). Esse padrão de distribuição, tendendo a J reverso evidencia um ótimo estado de conservação da área, ou seja, não há distúrbios comprometendo o número de indivíduos na primeira classe.

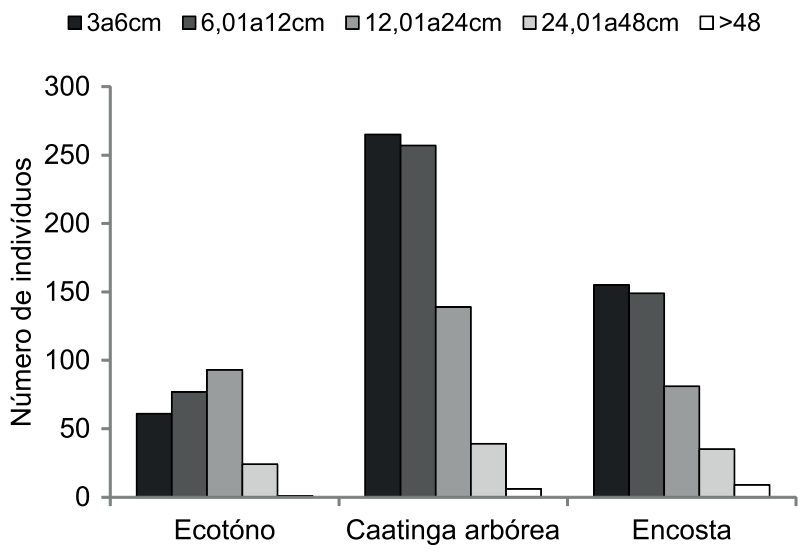

FIGURA 2 Distribuição de frequência em classes de diâmetro dos indivíduos arbóreos amostrados em três ambientes de Floresta Tropical Sazonalmente Seca no Parque Nacional Cavernas do Peruaçu.

FIGURE 2 Diameter classes frequency distribution of the trees sampled in the three environments of Seasonally Dry Tropical Forest at Cavernas do Peruaçu National Park.

\section{CONCLUSÕES}

Existem tanto diferenciações florísticas quanto estruturais entre encosta, Caatinga arbórea e ecótono com Cerrado. As diferenciações florísticas foram evidenciadas, tanto pelas espécies indicadoras quanto pelo grande número de espécies exclusivas a cada ambiente. Quanto às variações estruturais, - ecótono apresentou os menores valores tanto de densidade quanto de área basal. Já, a encosta e Caatinga arbórea apresentaram estratégias opostas, indivíduos encontrados na encosta apresentaram maior crescimento em biomassa, representado pela área basal, já, os indivíduos da Caatinga arbórea demonstraram maior investimento em reprodução, evidenciado pelo maio número de indivíduos. 


\section{REFERÊNCIAS}

ALCOFORADO-FILHO, F. G.; SAMPAIO, E. V. S. B.; RODAL, M. J. N. Florística e fitossociologia de um remanescente de vegetação caducifólia espinhosa arbórea em Caruaru, Pernambuco. Acta Botanica Brasilica, Porto Alegre, v. I7, n. 2, p. 287-303, 2003.

BRASIL. Decreto $s / n^{\circ}$, de 21 de setembro de 1999. Cria o Parque Nacional Cavernas do Peruaçu, no Estado de Minas Gerais, e dá outras providências. Diário Oficial [da] República Federativa do Brasil, Brasília, 22 set. 1999. Seção I, p. 6.

BRASIL. Ministério da Integração Nacional. Secretaria de Políticas de Desenvolvimento Regional . Nova delimitação do semi-árido brasileiro. Brasília, 2005.

BROWER, J. E.; ZAR, J. H. Field and laboratory methods for general ecology. Dubuque: W. M. C. Brow, 1984.

DUFRÊNE, M.; LEGENDRE, P. Species assemblages and indicator species: the need for a flexible asymmetrical approach. Ecological Monograhs, Washington, v. 67, p. 345-366, 1997.

GENTRY, A. H. Bignoniaceae: part II, tribe Tecomeae. Flora Neotropica, New York, v. 25, p. 370, 1992.

GOMES, F. T.; SILVA, E. P.; CORREA, J. B.; AVELINO, A. S. Plano operativo de prevenção e combate aos incêndios florestais no Parque Nacional Cavernas do Peruaçu. Itacarambi: IBAMA, 2007. Disponível em: <http://www.ibama.gov.br/phocadownload/category/44p?download $=2331>$. Acesso em: 10 nov. 2013 .

LINARES-PALOMINO, R.; OLIVEIRA-FILHO, A. T.; PENNINGTON, R. T. Neotropical seasonally dry forests: diversity, endemism and biogeography of woody plants. In: DIRZO, R.; YOUNG, H. S.; MOONEY, H. A.; CEBALLOS, G. (Ed.). Seasonally dry tropical forests: ecology and conservation. Washington: Island, 20I I. p. 3-21.

LORENZI, H. Árvores brasileiras: manual de identificação e cultivo de plantas arbóreas do Brasil. São Paulo: Plantarum, 1998. v. I, 352 p.

MARQUETE, R.; VAZ, A. M. S. O gênero Casearia no Estado do Rio de Janeiro, Brasil. Rodriguésia, Rio de Janeiro, v. 58, n. 4, p. 705-738, 2007.

MCCUNE, B.; MEFFORD, M. J. PC-ORD version 4.0, multivariate analysis of ecological data: users guide. Glaneden Beach: MjM Software Design, 1999. 237 p.

MEYER, H. A.; RECKNAGEL, A. B.; STEVENSON, D. D.; BARTOO, R. A. Forest management. $2^{\text {nd }}$ ed. New York: Ronald, I96I. 282 p.
MUELLER-DOMBOIS, D.; ELLEMBERG, H. Aims and methods of vegetation ecology. New York: J. Wiley, 1974.

OLIVEIRA, G.; ARAÚJO, M. B.; RANGEL, T. F.; ALAGADOR, D.; DINIZ-FILHO, J. A. F. Conserving the Brazilian semiarid (Caatinga) biome under climate change. Biodiversity and Conservation, Dordrecht, v. 2I, p. 29I3-2926, 2012.

OLIVEIRA FILHO, A. T.; RATTER, J. A. Padrões florísticos das Matas ciliares da Região do Cerrado e a evolução das paisagens do Brasil Central durante o Quaternário Tardio. In: RODRIGUES, R. R.; LEITÃO FILHO, H. F. (Ed.). Matas ciliares: conservação e recuperação. 2. ed. São Paulo: EDUSP; FAPESP, 2009. p. 73-89.

PENNINGTON, R. T.; LAVIN, M.; OLIVEIRA-FILHO, A. T. Evolution, and ecology in the tropics: perscpectives from seasonally dry tropical forests. Annual Review of Ecology, Evolution, and Systematics, Palo Alto, v. 40, p. 437-457, 2009.

PRADO, D. E.; GIBBS, P. E. Patterns of species distributions in the dry seasonal forest of South America. Annual of the Missouri Botanical Garden, Saint Louis, v. 80, p. 902927, 1993.

QUEIROZ, L. P. The Brazilian Caatinga: phytogeographical patterns inferred from distribution data of the Leguminosae. In: PENNINGTON, R. T.; RATTER, J. A.; LEWIS, G. P. (Ed.). Neotropical savannas and dry forests: plant diversity, biogeography and conservation. Boca Raton: CRC, 2006. p. I13- 149. (The Systematics Association Special volume Series, 69).

RODAL, M. J. N.; MARTINS, F. R.; SAMPAIO, V. S. B. E. Levantamento quantitativo das plantas lenhosas em trechos de vegetação de Caatinga em Pernambuco. Revista Caatinga, Mossoró, v. 21, n. 3, p. 192-205, 2008.

SANTOS, R. M.; BARBOSA, A. C. M. C.; ALMEIDA, H. S.; VIEIRA, F. A.; SANTOS, P. F.; CARVALHO, D. A.; OLIVEIRA-FILHO, A. T. Estrutura e florística de um remanescente de Caatinga Arbórea em Juvenília, Norte de Minas Gerais, Brasil. Cerne, Lavras, v. I7, n. 2, p. $247-$ 258,2011 .

SANTOS, R. M.; OLIVEIRA-FILHO, A. T.; EISENLOHR, P. V.; QUEIROZ, L. P.; CARDOSO, D. B. O. S.; RODAL, M. J. $\mathrm{N}$. Identity and relationships of the Arboreal Caatinga among other floristic units of seasonally dry tropical forests (SDTFs) of North-eastern and Central Brazil. Ecology and Evolution, Hoboken, v. 2, p. 409-428, 2012.

SANTOS, R. M.; VIEIRA, F. A. Análise estrutural do componente arbóreo de três áreas de cerrado em diferentes estádios de conservação no município de Três Marias, Minas Gerais, Brasil. Cerne, Lavras, v. II, n. 4, p. 399-408, dez. 2005. 
SANTOS, R. M.; VIEIRA, F. A.; FAGUNDES, M.; NUNES, Y. R. F; GUSMÃO, E. Riqueza e similaridade florística de oito remanescentes florestais no norte de Minas Gerais, Brasil. Revista Árvore, Viçosa, v. 31, n. I, p. I35-|44, 2007.

SILVA, L. A.; SCARIOT, A. Composição e estrutura da comunidade arbórea de uma Floresta Estacional Decidual sobre Afloramento calcário no Brasil Central. Revista Árvore, Viçosa, v. 28, n. I, p. 69-75, 2004.
TABARELLI, T.; SILVA, J. M. C. Áreas e ações prioritárias para a conservação da biodiversidade da caatinga. In: LEAL, I. R.; TABARELLI, M.; SILVA, J. M. C.; BARROS, M. L. B. (Ed.). Ecologia e conservação da caatinga. Recife: UFPE, 2003. p. 777-796.

VELLOSO, A. L.; SAMPAIO, E. V. S. B.; PAREYN, F. G. C. (Ed.). Ecorregióes: propostas para o bioma caatinga. Recife: Associação Plantas do Nordeste/Instituto de Conservação Ambiental The Nature Conservancy do Brasil, 2002. 
